\title{
Studying clusters and nano-precipitates in Aluminium alloys using SPED and ADF- STEM
}

Elisabeth Thronsen ${ }^{1}$, Adrian Lervik ${ }^{2}$, Christoph Hell ${ }^{3}$, Calin D. Marioara ${ }^{4}$, Sigurd Wenner ${ }^{5}$, Ruben Bjørge ${ }^{6}$, Jesper Friis ${ }^{5}$, SIgmund J. Andersen ${ }^{5}$ and Randi Holmestad ${ }^{3}$

${ }^{1}$ Norwegian university of science and technology, United States, ${ }^{2}$ NTNU, United States, ${ }^{3}$ NTNU, Trondheim, Sor-Trondelag, Norway, ${ }^{4}$ SINTEF Industry, United States, ${ }^{5}$ SINTEF, United States, ${ }^{6}$ SINTEF, Norway

Age-hardenable aluminium (Al) - Al-Cu, Al-Mg-Si and Al-Zn-Mg - alloys are important structural materials for construction and automotive applications due to properties like high strength/weight ratio and good formability. These properties are caused by small precipitates which are volume (3D) defects in the Al fcc host lattice. The crystal phases, morphologies and distributions of precipitates can be manipulated by chemical composition and thermo-mechanical treatment. Our research group at NTNU and SINTEF in Trondheim has for decades worked together with the Norwegian light metal industry on studies of precipitates and microstructure in these Al alloys [1]. Precipitation starts out with nanoscale clustering of solute elements and vacancies from a supersaturated, metastable solid solution in the Al host lattice. Solving the structure and quantifying the distribution of these clusters are important for understanding the further precipitation and thus the design of better alloys.

Two TEM techniques are used to study the clusters. Annular dark field scanning transmission electron microscopy (ADF-STEM) with different collection angles, using a double Cs corrected JEOL ARM200CF, gives information about atomic structure and lattice strain. STEM simulations and quantitative analysis of column intensities and spacings can enhance the information obtained from STEM imaging. The scanning (precession) electron diffraction (S(P)ED) technique can be used for structure determination of a large number of clusters for statistical and quantitative studies. [2]. Comparison between the unique PED patterns in the experimental data stack with simulated nanobeam diffraction patterns allows for verification or modification of the atomic model of the clusters initially deduced from STEM images. These models are subsequently assessed using density functional theory (DFT). The SPED experiments are performed on a JEOL 2100F equipped with a Quantum Detectors Merlin direct electron detector. Recently we have also started conducting quantitative convergent beam electron diffraction (QCBED) experiments of the clusters which gives promising results [3].

We have recently proposed the structure of the main clusters, the so-called GPI zones, in Al-Zn-Mg alloys [4] and found that they are composed of one fundamental basic unit. This is essentially a partial substitution by Mg and $\mathrm{Zn}$ on an fcc unit cell and a surrounding truncated cube octahedral (TCO) shell with a possible interstitial at the centre of the TCO. The TCO units arrange in certain directions according to simple principles to form larger clusters, as shown in figure 1. The virtual dark-field image obtained from a SPED data stack in figure 2 shows a fine dispersion of clusters in the Al-Zn-Mg alloy. Due to the small size of the clusters, the cluster/Al signal ratio in the diffraction patterns is low. To increase the signal, the diffraction patterns in all pixels belonging to a single cluster can be added, as shown in figure 2 . From the virtual dark-field image, we are investigating the possibility of extracting size and density of the clusters. In the Al-Mg-Si system, high angle ADF-STEM imaging is much more challenging because of the similar Z contrast from all atoms. Additionally, the clusters are also smaller and 
less developed for similar heat treatments. Due to their induced strain to the surrounding matrix, however, bright field TEM or low angle ADF-STEM imaging can be used to locate the clusters. We think S(P)ED combined with simulation of diffraction patterns will become a powerful technique for elucidating the structure of the clusters in the Al-Mg-Si system, which still remains unknown.

This presentation will show our last work in combining advanced analysis of HAADF-STEM and S(P)ED to get more information about the early-stage clusters in Al alloys. This work is supported by the Research Council of Norway, Hydro, Benteler and Neuman trough the SumAl (Solute cluster manipulation for optimized properties in Al-Mg-Si based Al alloys) project (NFR 294933), the Research Council of Norway via the NORTEM infrastructure (NFR 197405) and by Norwegian University of Science and Technology (NTNU) via the Digital Transformation initiative 'Alldesign'. 

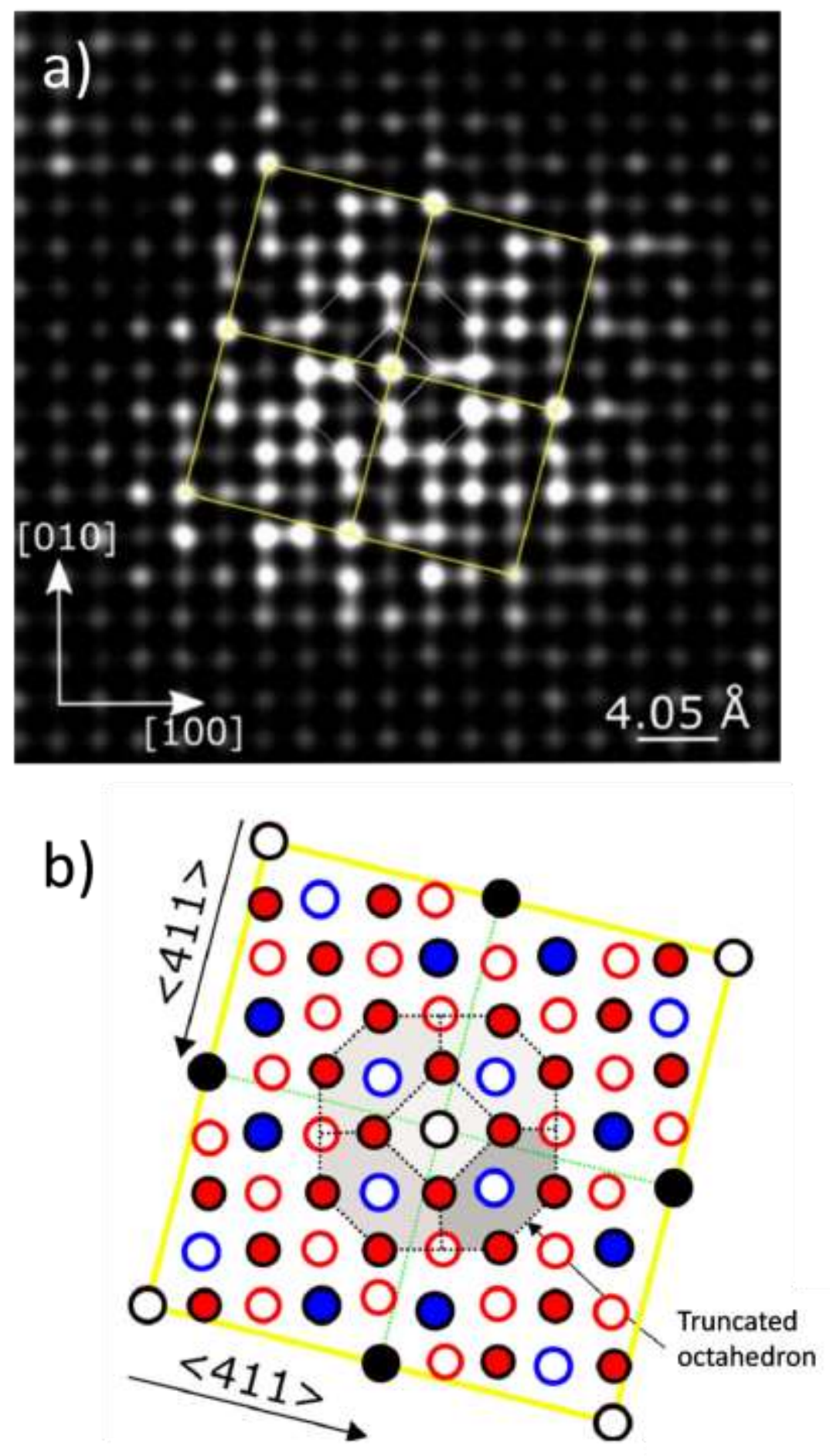

Figure 1. Figure 1: a) Filtered HAADF-STEM image of a cluster in an Al-Zn-Mg alloy. b) Corresponding suggested atomic column maps showing the $\langle 411\rangle$ connection principle between unit centres [4]. 

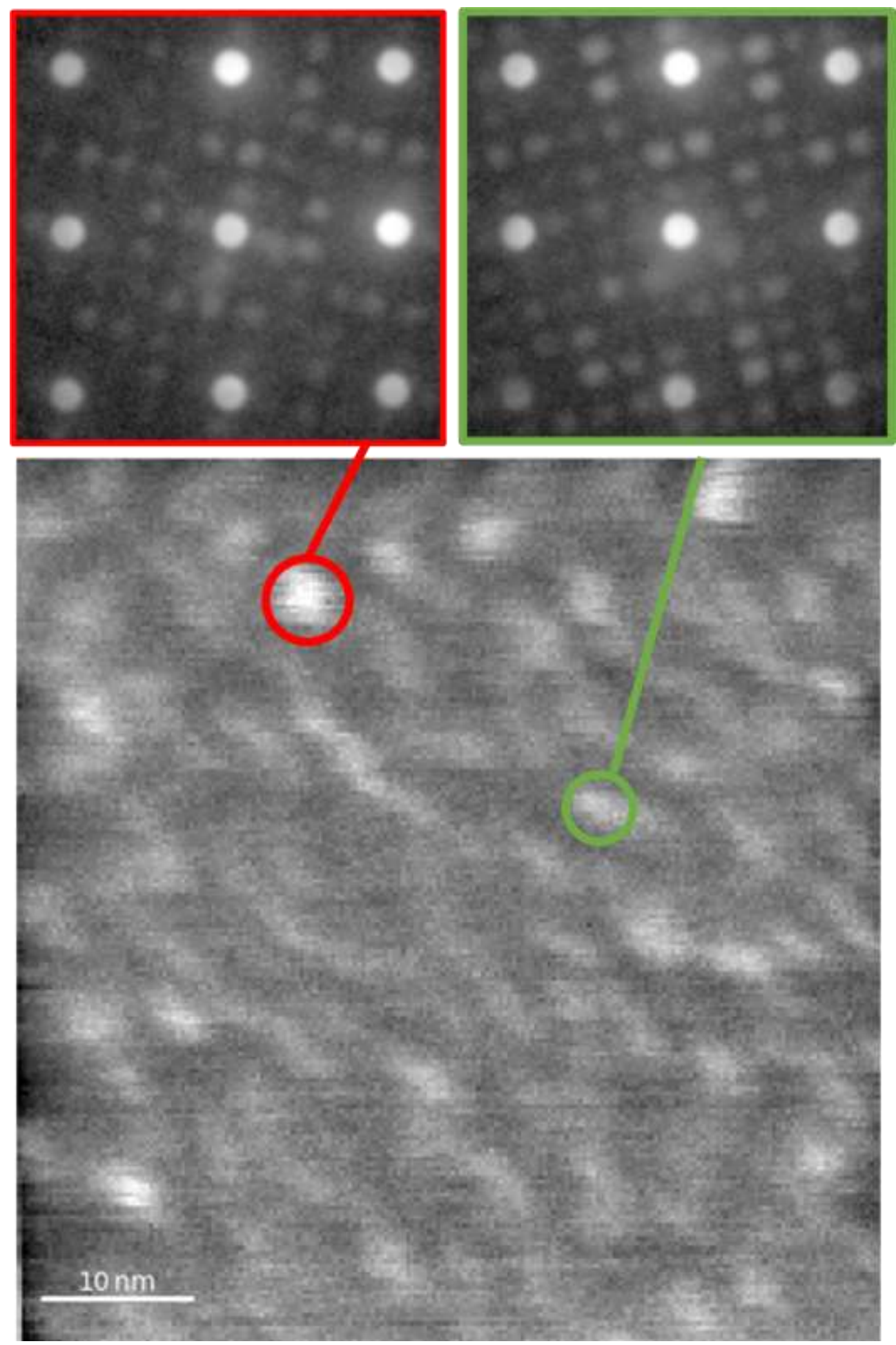

Figure 2. Figure 2: Virtual dark field image from clusters in an Al-Zn-Mg alloy made from a SPED data set. Diffraction patterns from two cluster areas are summed up to show diffraction patterns from the small clusters. 


\section{References}

[1] Andersen, S.J., Marioara, C.D., Friis, J., Wenner, S. \&Holmestad, R. Precipitates in aluminium alloys, Adv. in Phys. X, 3:1, 1479984 (2018), https://doi.org/10.1080/23746149.2018.1479984; Saito, T., Mørtsell, E.A., Wenner, S., Marioara, C.D., Andersen, S.J., Friis, J., Matsuda, K. \&Holmestad, R. Adv. Eng. Mater. 20, 1800125 (2018). https://doi.org/10.1002/adem.201800125.

[2] Sunde, J.K., Marioara, C.D., van Helvoort, A.T.J., Holmestad, R.The evolution of precipitate crystal structures in an Al-Mg-Si(-Cu) alloy studied by a combined HAADF-STEM and SPED approach, Mat. Char., 142, 458 (2018), https://doi.org/10.1016/j.matchar.2018.05.031.

[2] Thronsen et al. (2021), this proceeding.

[3] Lervik, A., Thronsen, E., Friis, J., Marioara, C.D., Wenner, S., Bendo, A., Matsuda, K., Holmestad, R. \& Andersen, S.J. Atomic structure of solute clusters in Al-Zn-Mg alloys. Acta Mat. 205, 116574 (2021). http://dx.doi.org/10.1016/j.actamat.2020.116574 